\title{
Lycopene Inhibits Proliferation, Invasion and Migration of Human Breast Cancer Cells
}

\author{
Min-Soo KoH ${ }^{a}$, Jin-Sun Hwang ${ }^{a}$, and Aree Moon* \\ College of Pharmacy, Duksung Women's University, Seoul 132-714, Republic of Korea
}

(Received January 18, 2010; Revised January 25, 2010; Accepted January 25, 2010)

\begin{abstract}
Breast cancer has been estimated as one of the most common causes of cancer death among women. The major cause of death from breast cancer is the metastatic spread of the disease from the primary tumor to distant sites in the body. Lycopene is one of the major carotenoids in fruits and vegetables including tomatoes. Epidemiological studies have shown that the dietary intake of lycopene is associated with decreased risk of cancer. Although mounting evidence shows the chemopreventive effect of lycopene, the role of lycopene in the prevention of metastatic potential of breast cancer has not been determined yet. In the present study, we investigated the inhibitory effect of lycopene on invasive and migratory phenotypes of two highly aggressive breast cancer cell lines, H-Ras-transformed MCF10A human breast epithelial cells (H-Ras MCF10A) and MDA-MB-231 human breast cancer cells. Here, we report that lycopene significantly inhibits invasion and migration as well as proliferation of H-Ras MCF10A and MDA-MB-231 cells. This study suggested an in vitro anti-cancer and anti-metastatic potential of lycopene. We also showed that activations of ERKs and Akt were inhibited by lycopene in H-Ras MCF10A cells, suggesting that the ERKs and Akt signaling pathways may be involved in lycopene-induced anti-proliferative and/or anti-invasive/migratory effects in these cells. Taken in conjunction with the fact that breast cancer metastasis is one of the most lethal malignancies in women, our findings may provide useful information for the application of lycopene in establishing strategy to prevent the metastatic breast cancer.
\end{abstract}

Keywords: Lycopene, H-Ras MCF10A, MDA-MB-231, Invasion, Breast cancer

\section{INTRODUCTION}

Breast cancer has been estimated as the most commonly diagnosed type of cancer and the second most common cause of cancer death among women (Jemal et al., 2007). Metastasis, a characteristic of highly malignant cancers with poor clinical outcome, has been one of the major causes of mortality in breast cancer patients. Thus, inhibition of the invasion and metastasis of cancer cells is of great significance in cancer treatment (Hwang and Lee, 2006). Since metastasis represents the most important cause of cancer death, anti-tumor agents that may inhibit this process have been extensively pursued. Metastasis of malignantly transformed cells is a multi-step process, which involves detachment of cells from the primary tumor,

*Corresponding author

Tel: +82-2-901-8394 Fax: +82-2-901-8386

E-mail: armoon@duksung.ac.kr

${ }^{\mathrm{a} B}$ Both of the authors contributed equally to this study. attachment to the extracellular matrix (ECM), degradation of the ECM components (invasion) and migration of cells through degraded matrix (Stetler-Stevenson et al., 1993). A role for members of matrix metalloproteinase (MMP) family on tumor invasion and metastasis has been suggested, especially, MMP-2 and MMP-9 (Liotta et al., 1979; DeClerck et al., 1992).

Elevated levels of the Ras protein are detected in $60-70 \%$ of human primary breast carcinomas (Clair et al., 1987 ) and Ras has been suggested as a marker of tumor aggressiveness in breast cancer. We previously showed that $\mathrm{H}$-Ras, but not $\mathrm{N}$-Ras, induced invasive and migratory phenotypes in MCF10A human breast epithelial cells (Moon et al., 2000). We also showed that H-Ras-induced invasiveness was associated with the activation of p38 mitogen-activated protein kinase (MAPK) and extracellular signal-regulated kinases (ERKs), resulting in induction of MMP-9 and MMP-2 (Kim et al., 2003; Shin et al., 2005; Song et al., 2006). 
Chemoprevention is arguably one of the major weapons in the anticancer arsenal (Ferguson, 1994; Stavric, 1994). Efforts have been made to develop a chemoprevention strategy that can inhibit proliferation of malignant cancer cells. There has been a growing body of evidence that phytochemicals in our diet can reduce the risk of cancers. Cancer chemoprevention has emerged as a new strategy against cancer, using of pharmacologic or natural agents that inhibit the development of invasive cancer (Kim and Moon, 2004).

Carotenoids are important phytochemicals that are known for the protective effects of fruits and vegetables (Rao and Agarwal, 1999). Epidemiological studies reported that dietary intake of a carotenoid lycopene is associated with decreased risk of cancer (Franceschi et al., 1994; Giovannucci, 1999; Agarwal and Rao, 2000). Lycopene is the pigment principally responsible for the bright red color found in ripe tomatoes (Lycopersicon esculentum) and tomato products (Omoni and Aluko, 2005). The structure of lycopene is a lipophilic, 40-carbon atom, highly unsaturated, straight chain hydrocarbon containing 11 conjugated and 2 non- conjugated double bonds as depicted in Fig. 1 (Boileau et al., 1999; Rao and Agarwal, 2000; Shi, 2000). The 11 conjugated double bonds of lycopene make it a potentially powerful anti-oxidant and lycopene has most anti-oxidant effect among carotenoids (Stahl and Sies, 1996; Arab and Steck, 2000). Epidemiological studies have demonstrated that high consumption of lycopene reduces the risk of breast and other types of human cancers (Rao and Agarwal, 1999; Weisburger, 2002; Wu et al., 2003). Giovannucci (1999) reported that intake of tomatoes and tomato-based products and plasma levels of lycopene have been relatively consistently associated with a lower risk of a variety of cancers. Several in vitro studies have demonstrated the anti-proliferation property of lycopene in endometrial, mammary, lung, and prostate human cancer cells (Levy et al., 1995; Kim et al., 2002). In an in vivo study, lycopene-fed mice had delayed onset and reduced spontaneous mammary tumor growth and development (Nagasawa et al., 1995). Recently, it has been demonstrated that lycopene reduced experimental tumor metastasis and such an action was associated with

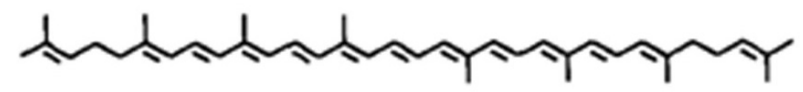

Fig. 1. Structure of all-trans lycopene which is the most prominent isomer found in fresh tomatoes (Cunningham et al., 1994). attenuation of proliferation, invasion and angiogenesis of hepatoma cells (Huang et al., 2008).

In the present study, we attempted to investigate the in vitro chemopreventive effect of lycopene and the possible mechanism of action on two highly aggressive breast cancer cell lines, $\mathrm{H}$-Ras-transformed MCF10A human breast epithelial cells (H-Ras MCF10A) and MDA-MB-231 human breast cancer cells. Here, we report that lycopene inhibits proliferation of H-Ras MCF10A and MDA-MB-231 cells. We also provide evidence that lycopene reduces invasive and migratory properties of breast cancer cells, suggesting a possible application of lycopene for prevention of metastatic breast cancer.

\section{MATERIALS AND METHODS}

\section{Cell lines and culture condition}

$\mathrm{H}$-Ras MCF10A cells were established as previously described (Moon et al., 2000). H-Ras MCF10A cells were maintained at $37^{\circ} \mathrm{C}$ in a humidified atmosphere of $5 \%$ $\mathrm{CO}_{2} / 95 \%$ air in DMEM/F12 medium supplemented with $5 \%$ heat-inactivated horse serum, $10 \mu \mathrm{g} / \mathrm{ml}$ insulin, 100 $\mathrm{ng} / \mathrm{ml}$ cholera toxin, $0.5 \mu \mathrm{g} / \mathrm{ml}$ hydrocortisone, $20 \mathrm{ng} / \mathrm{ml}$ recombinant EGF, $2 \mathrm{mM}$ L-glutamine, and $100 \mu \mathrm{g} / \mathrm{ml}$ penicillin-streptomycin and $0.5 \mu \mathrm{g} / \mathrm{ml}$ fungizone. MDA-MB-231 cells were kindly provided by Dr. Dong Young Noh (Seoul National University, Seoul, Korea) and cultured in DMEM supplemented with $10 \%$ FBS and $100 \mu \mathrm{g} / \mathrm{ml}$ penicillinstreptomycin. The cells were maintained in humidified atmosphere with $95 \%$ air and $5 \% \mathrm{CO}_{2}$ at $37^{\circ} \mathrm{C}$.

\section{Preparation of lycopene}

Lycopene (99\%) was purchased from Sigma Chemical (St. Louis, MI). Lycopene was dissolved in tetrahydrofuran (THF) containing $0.25 \%$ butylated hydroxytoluene (BHT). Stock solution was prepared under the minimum of exposure to air and light and stored at $-70^{\circ} \mathrm{C}$. Immediately before the experiment, aliquots from the stock solution were added to the cell culture medium, and the final concentration of lycopene was ranged from $0.1 \mu \mathrm{M}$ to $20 \mu \mathrm{M}$. All procedures including cell treatment were carried out under dim light and on the ice. The concentration of THF in the media was $0.25 \%$, which did not cause cytotoxicity (data not shown).

\section{3-(4, 5-dimethylthiazol-2-yl)-2, 5-diphenyl-tetrazolium bromide (MTT) assay}

Cells $\left(5 \times 10^{3}\right)$ cultured in a 96-well plate were treated with lycopene for $24 \mathrm{hr}$. After $24 \mathrm{hr}$ of incubation, $25 \mu \mathrm{l}$ of $0.5 \mathrm{mg} / \mathrm{ml}$ of MTT was added and incubated for $4 \mathrm{hr}$. 
Conversion of MTT into purple formazan by metabolically active cells indicates the extent of cell viability. The crystals of produced formazan were dissolved with $100 \mu$ of DMSO and the optical density was measured at $540 \mathrm{~nm}$ using a micro-ELISA reader (Molecular Devices, Sunnyvale, CA) for quantification of cell viability. Assays were performed triplicate.

\section{In vitro invasion assay}

In vitro invasion assay was performed using 24-well transwell unit with polycarbonate filters (Corning Costar, Cambridge, MA) as previously described (Kim et al., 2003). The lower side of the filter was coated with type I collagen, and the upper side was coated with Matrigel (Collaborative Research, Lexington, KY). Lower compartment was filled with serum-free media containing $0.1 \%$ BSA. Cells were placed in the upper part of the Transwell plate, incubated for $17 \mathrm{hr}$, fixed with methanol and stained with hematoxylin for $10 \mathrm{~min}$ followed briefly by eosin. The cells on the upper surface of the filter were removed using a cotton swab. The invasive phenotypes were determined by counting the cells that invaded to the lower side of the filter with microscopy at $\times 400$. Thirteen fields were counted for each filter and each sample was assayed in triplicate.

\section{Transwell migration assay}

In vitro migration assay was performed using a 24 well transwell unit with polycarbonate filters as previously described (Kim et al., 2003). Experimental procedures were the same as the in vitro invasion assay described above except that the filter was not coated with Matrigel for the migration assay.

\section{Western blot analysis}

Western blot analysis was performed as previously described (Shin et al., 2005). Protein extracts in lysis buffer (0.5\% Triton X-100, 0.15M NaCl, $50 \mathrm{mM}$ Tris- $\mathrm{HCl}, \mathrm{pH} 7.4$, $25 \mathrm{mM} \mathrm{NaF}, 20 \mathrm{mM}$ EGTA, $1 \mathrm{mM}$ DTT, $1 \mathrm{mM} \mathrm{Na}_{3} \mathrm{VO}_{4}$ ) containing protease inhibitor cocktail (Roche, Mannheim, Germany) were subjected to $12 \%$ SDS-PAGE analysis and transferred to nitrocellulose membrane. The levels of $\mathrm{Bcl}-2$ and Bax were detected using anti-Bcl-2 (DAKO, Denmark) and anti-Bax (Santa Cruz Biotechnology Inc., Santa Cruz, CA, USA) antibodies. Anti-ERK, anti-phosphorylated ERK, anti-p38, anti-phosphorylated p38, anti-Akt, anti-phosphorylated Akt, anti-SAPK/JNK, anti-phosphorylated-SAPK/JNK were purchased from Cell Signaling Technology, Inc. (Beverly, MA, USA). Enhanced chemiluminescence (ECL, Amersham-Pharmacia) system was used for detection. Relative band intensities were determined by quantitation of each band with an Image Analyzer (Vilber Lourmet).

\section{RESULTS AND DISCUSSION}

A dose response study was conducted to examine the effect of lycopene on the growth of breast cancer cell lines. Treatment with lycopene for $24 \mathrm{hr}$ inhibited the growth of $\mathrm{H}$-Ras MCF10A cells in a dose-dependent manner with the $\mathrm{IC}_{50}$ value of $1.3 \mu \mathrm{M}$ (Fig. 2, open circle). Following 24 $\mathrm{hr}$ of treatment with lycopene, the proliferation of MDA-MB-231 cells was also decreased with the $\mathrm{IC}_{50}$ value of $13.4 \mu \mathrm{M}$ (Fig. 2, closed circle). Lycopene was more effective in inhibiting proliferation of H-Ras MCF10A cells compared to that of MDA-MB-231 cells. Consistent with our results, it has previously been reported that lycopene inhibited the growth of MCF-7 and MDA-MB-231 breast cancer cells (Prakash et al., 2001).

Numerous natural products have been shown to exert growth-inhibitory effects on malignant cells through induction of apoptosis. It has been shown that lycopene exerts anti-proliferative effect on human colon carcinoma cells, human prostate carcinoma cells and a prototype of Burkitt lymphoma cells by induction of apoptosis (Hwang and Bowen, 2004; Salman et al., 2007). We then investigated if the anti-proliferative effect of lycopene involves

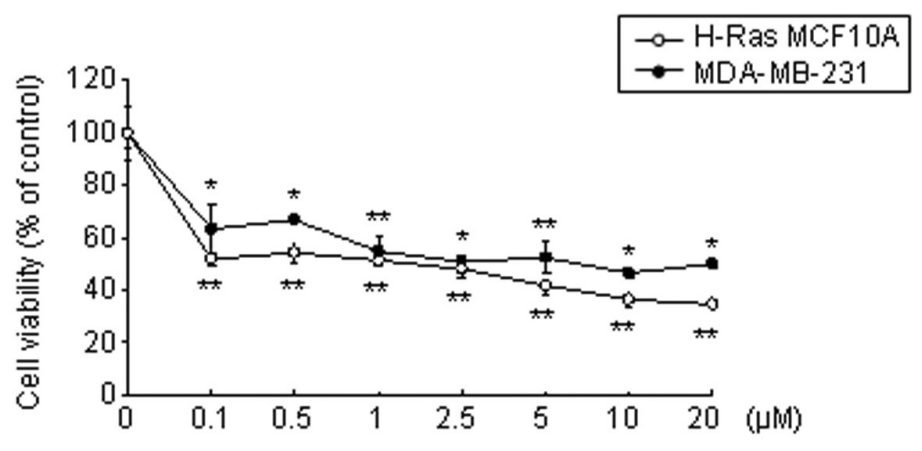

Fig. 2. Effects of lycopene on the growth of H-Ras MCF10A cells and MDA-MB-231 cells. Cells were treated with various concentrations of lycopene for $24 \mathrm{hr}$ and subjected to MTT assay. The results represent means \pm SE of triplicates. *, ${ }^{* *}$ Statistically different from control at $p<0.05$ and $p<0.01$, respectively. 
A

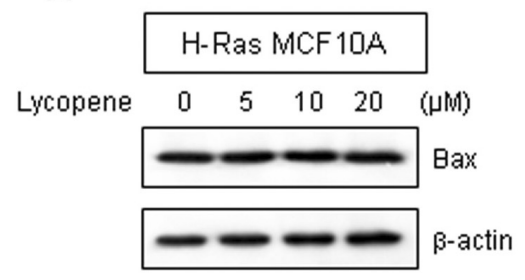

B

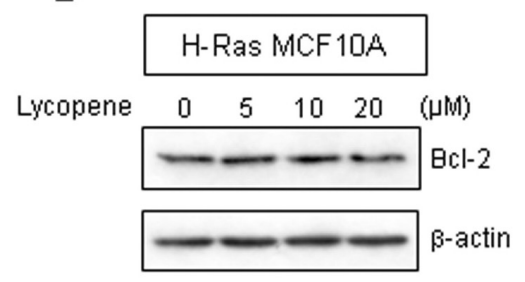

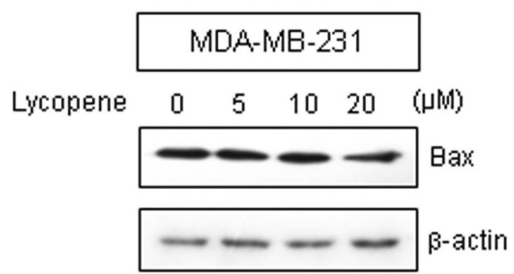

MDA-MB-231

Lycopene

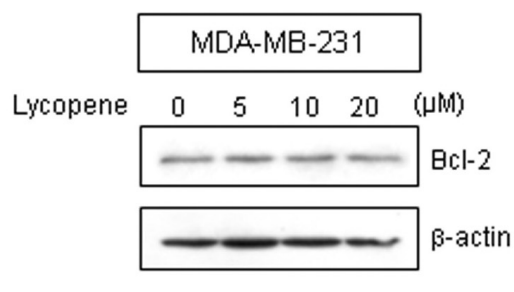

Fig. 3. Effects of lycopene on the expression of $\mathrm{Bcl}-2$ and Bax. H-Ras MCF10A cells and MDA-MB-231 cells were treated with various concentrations of lycopene for $24 \mathrm{hr}$. Western blot analysis was performed on cell lysates using anti-Bax antibody (A) and anti-Bcl-2 antibody (B). $\beta$-actin was used as a loading control.

A

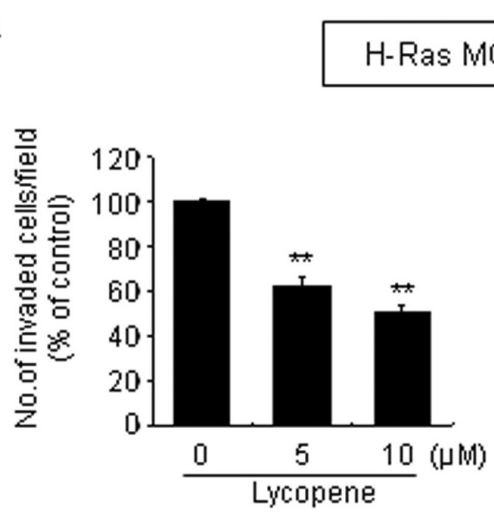

B

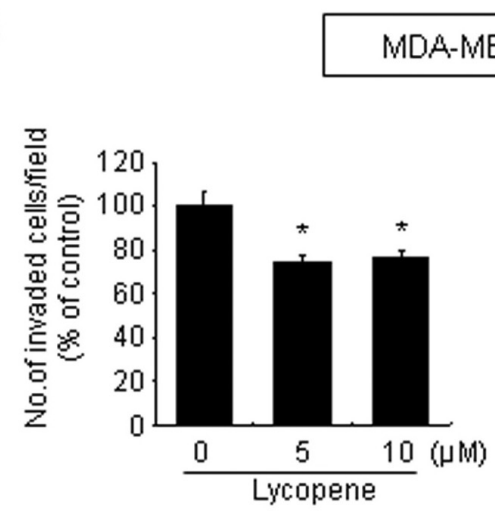

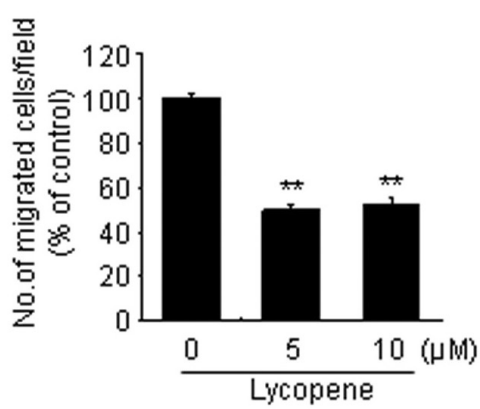


may not be due to induction of Bax or Bcl-2-related apoptotic cell death.

Invasive and migratory properties of cells are considered crucial in metastatic process of cancer. While many studies support the anti-proliferative effect of lycopene in a variety of cancers, there has been a limited amount of information on the anti-invasive and anti-metastatic effects of lycopene. A recent report showed that lycopene inhibited migration of platelet derived growth factor (PDGF)BB-induced human Hs68 skin fibroblast (Chiang et al., 2007). Lycopene significantly inhibited the invasive ability of hepatoma cells (Huang et al., 2007). Lycopene supplementation in vivo markedly inhibited the growth of metastatic tumors with attenuation of proliferation, invasion and angiogenesis in hepatoma cells (Huang et al., 2008). In this study, we investigated the effects of lycopene on the invasive and migratory phenotypes of human breast cancer cells. Treatment with $10 \mu \mathrm{M}$ of lycopene for $17 \mathrm{hr}$ significantly inhibited invasion and migration of $\mathrm{H}$-Ras MCF10A cells by $50 \%$ and $48 \%$, respectively (Fig. $4 \mathrm{~A}$ ). Invasive and migratory properties of MDA-MB-231 cells were also inhibited by $10 \mu \mathrm{M}$ of lycopene by $24 \%$ and $37 \%$, respectively (Fig. 4B). MMP-2 and/or MMP-9 have been shown to be responsible for the induction of invasive phenotype in many cell systems including breast cells (Kim et al., 2003; Song et al., 2006; Kim et al., 2010). We next examined if lycopene down-regulated MMP-2/-9 in H-Ras MCF10A and MDA-MB-231 cells by gelatin zymogram assay. Neither MMP-2 nor MMP-9 was affected upon lycopene treatment (data not shown).

In order to investigate the association of signaling molecules in lycopene-induced inhibition of proliferation and/or invasiveness of breast cancer cell lines, we examined the effect of lycopene on the activation of signaling molecules; ERK1/2, p38, c-Jun N-terminal kinase (JNK) and Akt. A kinetic study was conducted on H-Ras MCF10A and MDA-MB-231 cells to examine the activations of these signaling molecules upon treatment with $20 \mu \mathrm{M}$ lycopene. As shown in Fig. 5, phosphorylated forms of ERK1/2 and Akt were significantly reduced after exposure of lycopene in $\mathrm{H}$-Ras MCF10A cells while activation of p38 or JNK was not altered. These data suggest that activation of ERKs and Akt signaling pathways may be involved in lycopene-induced anti-proliferative and/or anti-invasive/migratory effects in H-Ras MCF10A cells. Consistent with our results, a recent study showed that lycopene inhibited
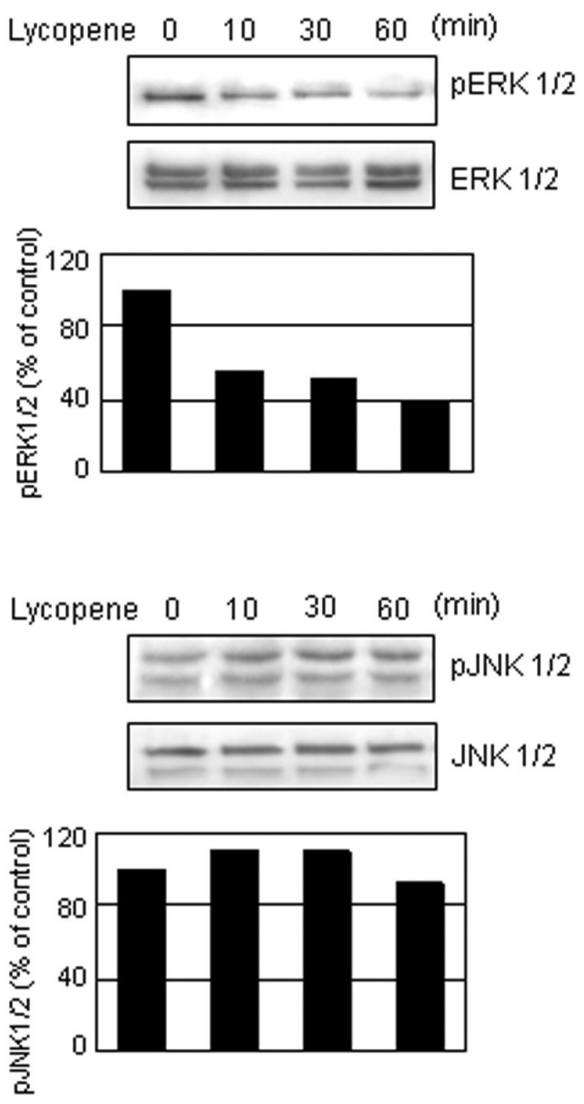
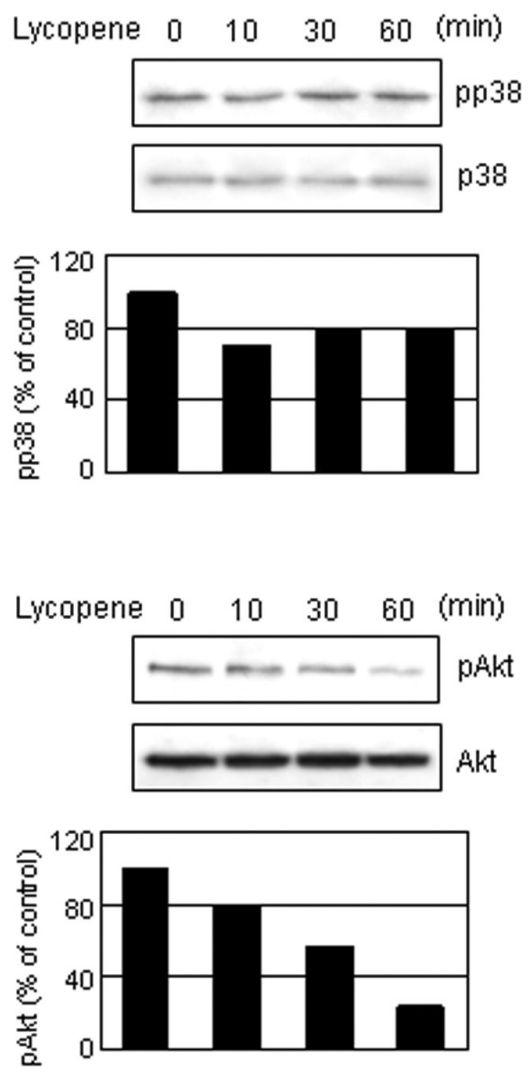

Fig. 5. Effects of lycopene on the phosphorylation of ERK1/2, p38, JNK and Akt in H-Ras MCF10A cells. Cells were treated with $20 \mu \mathrm{M}$ lycopene for indicated time points. Western blot analysis was performed using antibodies against phosphorylated or total forms of ERK1/2, p38, JNK and Akt. Band intensities were quantitated by densitometric measurements and the phosphorylated forms of ERK1/2, p38, JNK and Akt were plotted. 
PDGF-BB-induced migration of retinal pigment epithelial cell by suppression of PI3K/Akt and ERK1/2 pathways (Chan et al., 2009). In MDA-MB-231 cells, however, neither ERK1/2 nor Akt was significantly inhibited by lycopene (data not shown). Since lycopene is one of the members of vitamin A family compounds (Cunningham et al., 1994; Freemantle et al., 2003), a possible mechanism for the anti-proliferative, anti-invasive, and anti-migratory effects of lycopene may involve the retinoid receptor signaling. Further studies would be required to elucidate the molecular mechanism for the inhibitory effect of lycopene in proliferation and invasion/migration of breast cancer cells.

Taken together, the present study demonstrated that lycopene inhibited proliferation, invasion and migration of highly invasive breast cancer cell lines, H-Ras MCF10A and MDA-MB-231 cells. Given that breast cancer metastasis is one of the most lethal malignancies in women, our findings may provide useful information for the application of lycopene in establishing strategy to prevent the metastatic breast cancer.

\section{ACKNOWLEDGMENTS}

This research was supported by the Duksung Women's University Research Grant 2008.

\section{REFERENCES}

Agarwal, S. and Rao, V. A. (2000). Tomato lycopene and its role in human health and chronic diseases. CMJA. 163, 739-744.

Arab, L. and Steck, S. (2000). Lycopene and cardiovascular disease. Am. J. Clin. Nutr. 71 Suppl, 1691S-1695S.

Boileau, A. C., Merchen, N. R., Wasson, K., Atkinson, C. A. and Erdman, J. W. (1999). Cis-lycopene is more bioavailable than trans-lycopene in vitro and in vivo in lymph-cannulated ferrets. J. Nutr. 129, 1176-1181.

Chan, C. M., Fang, J. Y., Lin, H. H., Yang, C. Y. and Hung, C. F. (2009). Lycopene inhibits PDGF-BB-induced retinal pigment epithelial cell migration by suppression of PI3K/Akt and MAPK pathways. Biochem. Biophys. Res. Commun. 388, 172-176.

Chiang, H. S., Wu, W. B., Fang, J. Y., Chen, D. F., Chen, B. H., Huang, C. C., Chen, Y. T. and Hung, C. F. (2007). Lycopene inhibits PDGF-BB-induced signaling and migration in human dermal fibroblasts through interaction with PDGF-BB. Life Sci. 81, 1509-1517.

Clair, T., Miller, W. and Cho-Chung, Y. (1987). Prognostic significance of the expression of the ras protein with a molecular weight of 21,000 by human breast cancer. Cancer Res. 49, 5290-5293.

Cunningham, F. X. Jr., Sun, Z., Chamovitz, D., Hirschberg, J. and Gantt, E. (1994). Molecular structure and enzymatic function of lycopene cyclase from the Cyanobacterium Synechococcus sp Strain PCC7942. Plant. Cell. 6, 11071121.
DeClerck, Y. A., Perez, N., Shimada, H., Boone, T. C., Langley, K. E. and Taylor, S. M. (1992). Inhibition of invasion and metastasis in cells transfected with an inhibitor of metalloproteinases. Cancer Res. 52, 701-708.

Ferguson, L. R. (1994). Antimutagens as cancer chemopreventive agents in the diet. Mut. Res. 307, 395-410.

Franceschi, S., Bidoli, E., LaVeccia, C., Talamini, R., D'Avanzo, B. and Negri, E. (1994). Tomatoes and risk of digestive tract cancers. Int. J. Cancer 59, 181-184.

Freemantle, S. J., Spinella, M. J. and Dmitrovsky, E. (2003). Retinoids in cancer therapy and chemoprevention: promise meets resistance. Oncogene 22, 7305-7315.

Giovannucci, E. (1999). Tomatoes, tomato-based products, lycopene, and cancer: review of the epidemiologic literature. J. Natl. Cancer Inst. 91, 317-331.

Huang, C. S., Fan, Y. E., Lin, C. Y. and Hu, M. L. (2007). Lycopene inhibits matrix metalloproteinase-9 expression and down-regulates the binding activity of nuclear factor-kappa $B$ and stimulatory protein-1. J. Nutr. Biochem. 18, 449-456.

Huang, C. S., Liao, J. W. and Hu, M. L. (2008). Lycopene inhibits experimental metastasis of human hepatoma SKHep-1 cells in athymic nude mice. J. Nutr. 138, 538-543.

Hwang, E. S. and Bowen, P. E. (2004). Cell cycle arrest and induction of apoptosis by lycopene in LNCaP human prostate cancer cells. J. Med. Food 7, 284-289.

Hwang, E. S. and Lee, H. J. (2006). Inhibitory effects of lycopene on the adhesion, invasion, and migration of SK-Hep1 human hepatoma cells. Exp. Biol. Med. 231, 322-327.

Jemal, A., Siegel, R., Ward, E., Murray, T., Xu, J. and Thun, M. J. (2007). Cancer Statistics. CA Cancer J. Clin. 57, 43-66.

Kim, E. S., Jeong, J. B., Kim, S., Lee, K. M., Ko, E., Noh, D. Y., Hwang, K. T., Ha, J. H., Lee, C. H., Kim, S. G. and Moon, A. (2010). The G12 family proteins upregulate matrix metalloproteinase-2 via p53 leading to human breast cell invasion. Breast Cancer Res. Treat. [Epub ahead of print].

Kim, I. Y. and Moon, A. (2004). Inhibition of invasive phenotype and induction of apoptosis in human breast cancer cells by chemopreventive agents. J. Korean Assoc. Cancer Prev. 9, 59-67.

Kim, L., Rao, A. V. and Rao, L. G. (2002). Effect of lycopene on prostate LNCaP cancer cells in culture. J. Med. Food 5, 181-187.

Kim, M. S., Lee, E. J., Kim, H. R. and Moon, A. (2003). p38 kinase is a key signaling molecule for $\mathrm{H}$-Ras-induced cell motility and invasive phenotype in human breast epithelial cells. Cancer Res. 63, 5454-5461.

Levy, J., Bosin, E., Feldman, B., Giat, Y., Miinster, A., Danilenko, M. and Sharoni, Y. (1995). Lycopene is a more potent inhibitor of human cancer cell proliferation than either $\alpha$-carotene or $\beta$-carotene. Nutr. Cancer 24, 257-266.

Liotta, L. A., Abe, S., Robey, P. G. and Martin, G. R. (1979). Preferential digestion of basement membrane collagen by an enzyme derived from a metastatic murine tumor. Proc. Natl. Acad. Sci. U.S.A. 76, 2268-2272.

Moon, A., Kim, M. S., Kim, T. G., Kim, S. H., Kim, H. E., Chen, Y. Q. and Kim, H. R. (2000). H-ras, but not $\mathrm{N}$-ras, induces an invasive phenotype in human breast epithelial cells: a role for MMP-2 in the H-ras-induced invasive phenotype. Int. J. Cancer 85, 176-181.

Nagasawa, H., Mitamura, T., Sakamoto, S. and Yamamoto, K. 
(1995). Effects of lycopene on spontaneous mammary tumor development in SHN virgin mice. Anticancer Res. 15, 11731178

Omoni, O. A. and Aluko, E. R. (2005). The anti-carcinogenic and anti-atherogenic effects of lycopene: a review. Trends Food Sci. Technol. 16, 344-350.

Prakash, P., Russell, R. M. and Krinsky, N. I. (2001). In vitro inhibition of proliferation of estrogen-dependent and estrogen-independent human breast cancer cells treated with carotenoids or retinoids. J. Nutr. 131, 1574-1580.

Rao, A. V. and Agarwal, S. (1999). Role of lycopene as antioxidant carotenoid in the prevention of chronic diseases: a review. Nut. Res. 19, 305-323.

Rao, A. V. and Agarwal, S. (2000). Role of anti-oxidant lycopene in cancer and heart disease. J. Am. Coll. Nutr. 19, 563-569.

Salman, H., Bergman, M., Djaldetti, M. and Bessler, H. (2007) Lycopene affects proliferation and apoptosis of four malignant cell lines. Biomed. Pharmacother. 61, 366-369.

Shi, J. (2000). Lycopene in tomatoes: chemical and physical properties affected by food processing. Crit. Rev. Food Sci. Nutr. 40, 1-42.

Shin, I., Kim, S., Song, H., Kim, H. R. and Moon, A. (2005). H-Ras-specific activation of Rac-MKK3/6-p38 pathway: its critical role in invasion and migration of breast epithelial cells. J. Biol. Chem. 280, 14675-14683.

Song, H., Ki, S. H., Kim, S. G. and Moon, A. (2006). Activating transcription factor 2 mediates matrix metalloproteinase-2 transcriptional activation induced by p38 in breast epithelial cells. Cancer Res. 66, 10487-10496.

Stahl, W. and Sies, H. (1996). Lycopene: a biologically important carotenoid for humans? Arch. Biochem. Biophys. 336 $1-9$

Stavric, B. (1994). Role of chemopreventers in human diet Clinical Biochem. 27, 319-332.

Stetler-Stevenson, W. G., Aznavoorian, S. and Liotta, L. A. (1993). Tumor cell interactions with the extracellular matrix during invasion and metastasis. Annu. Rev. Cell Biol. 9, 541-557.

Weisburger, J. H. (2002). Lycopene and tomato products in health promotion. Exp. Biol. Med. 227, 924-927.

Wu, K., Schwatz, S. J., Platz, E. A., Clinton, S. K., Erdman, J. W., Ferruzzi, M. G., Willett W. C. and Giovannucci E. L. (2003). Variations in plasma lycopene and specific isomers over time in a cohort of US men. J. Nutr. 133, 1930-1936.

Yang, E. and Korsmeyer, S. J. (1996). Molecular thanatopsis: a discourse on the BCL2 family and cell death. Blood. 88, 386-401. 